Epidemiology and Infection

cambridge.org/hyg

\section{Original Paper}

*These authors contributed equally to this work

Cite this article: Wang $L$, Yang J, Chen L, Wang $\mathrm{W}, \mathrm{Yu} \mathrm{F}$, Xiong $\mathrm{H}$ (2022). Whole-genome sequencing of Mycobacterium tuberculosis for prediction of drug resistance. Epidemiology and Infection 150, e22, 1-8. https://doi.org/ $10.1017 /$ S095026882100279X

Received: 26 August 2021

Revised: 20 December 2021

Accepted: 22 December 2021

\section{Key words:}

Drug resistance; prediction; sensitivity; specificity; tuberculosis; whole-genome sequencing

\section{Author for correspondence:}

Fangyou Yu, E-mail: wzjxufu@163.com; Haiyan Xiong, E-mail: haiyanxiong@fudan.edu.

(c) The Author(s), 2022. Published by Cambridge University Press. This is an Open Access article, distributed under the terms of the Creative Commons AttributionNonCommercial-ShareAlike licence (http:// creativecommons.org/licenses/by-nc-sa/4.0), which permits non-commercial re-use, distribution, and reproduction in any medium, provided the same Creative Commons licence is used to distribute the re-used or adapted article and the original article is properly cited. The written permission of Cambridge University Press must be obtained prior to any commercial use.

\section{CAMBRIDGE UNIVERSITY PRESS}

\title{
Whole-genome sequencing of Mycobacterium tuberculosis for prediction of drug resistance
}

\author{
Luqi Wang ${ }^{1, \star}$, Jinghui Yang ${ }^{2, \star}$, Liang $\mathrm{Chen}^{3}$, Weibing Wang ${ }^{1,4}$, Fangyou $\mathrm{Yu}^{2,4}$ \\ and Haiyan Xiong1,4 (D) \\ ${ }^{1}$ Department of Epidemiology, School of Public Health, Fudan University, Shanghai 200032, China; ${ }^{2}$ Tuberculosis \\ Microbiology Laboratory, Shanghai Pulmonary Hospital, Shanghai 200082, China; ${ }^{3}$ Hackensack-Meridian Health \\ Center for Discovery and Innovation, Nutley, NJ, USA and ${ }^{4}$ School of Public Health, Fudan University, Key \\ Laboratory of Public Health Safety, Ministry of Education, Shanghai, China
}

\begin{abstract}
Whole-genome sequencing (WGS) has shown tremendous potential in rapid diagnosis of drug-resistant tuberculosis (TB). In the current study, we performed WGS on drug-resistant Mycobacterium tuberculosis isolates obtained from Shanghai $(n=137)$ and Russia $(n=78)$. We aimed to characterise the underlying and high-frequency novel drug-resistance-conferring mutations, and also create valuable combinations of resistance mutations with high predictive sensitivity to predict multidrug- and extensively drug-resistant tuberculosis (MDR/XDR-TB) phenotype using a bootstrap method. Most strains belonged to L2.2, L4.2, L4.4, L4.5 and L4.8 lineages. We found that WGS could predict $82.07 \%$ of phenotypically drug-resistant domestic strains. The prediction sensitivity for rifampicin (RIF), isoniazid (INH), ethambutol (EMB), streptomycin (STR), ofloxacin (OFL), amikacin (AMK) and capreomycin (CAP) was $79.71 \%, 86.30 \%, 76.47 \%, 88.37 \%, 83.33 \%, 70.00 \%$ and $70.00 \%$, respectively. The mutation combination with the highest sensitivity for MDR prediction was $r p o B$ S450L $+r p o B$ $\mathrm{H} 445 \mathrm{~A} / \mathrm{P}+$ katG S315T + inhA I21T + inhA S94A, with a sensitivity of $92.17 \%(0.8615$, 0.9646 ), and the mutation combination with highest sensitivity for XDR prediction was $r p o B$ S450L + katG S315T + gyrA D94G + rrs A1401G, with a sensitivity of $92.86 \%(0.8158$, 0.9796). The molecular information presented here will be of particular value for the rapid clinical detection of MDR- and XDR-TB isolates through laboratory diagnosis.
\end{abstract}

\section{Introduction}

Drug-resistant tuberculosis (TB) has been a serious obstacle for global TB control programmes. TB patients with drug resistance may be induced by exposure to multidrug- and extensively drug-resistant tuberculosis (MDR/XDR-TB) strains or may develop as a result of other clinical factors, including delayed diagnosis, inappropriate treatment or poor compliance [1]. Controlling the high prevalence of drug-resistant TB largely depends on a timely laboratory diagnosis. Traditional TB drug susceptibility testing (DST) relies on solid or liquid culture, which may take weeks or months to yield results. The slow growth of Mycobacterium tuberculosis is an impediment to the rapid diagnosis of anti-TB drug resistance, and aggravates the situation by increasing the incidence of MDR and XDR-TB in the world. Some rapid molecular biology-based diagnostic methods have recently been applied in a clinical setting, including Xpert MTB/RIF and GenoType MTBDRplus [2-4]. Although these methods are rapid and simple, their extension to undeveloped areas of the world is limited by prohibitive costs and availability.

Whole-genome sequencing (WGS), as a molecular diagnostic tool, has been greatly developed in TB research since the first complete genome sequence H37Rv was announced in 1998. The sensitivity and specificity reported for predicting single common anti-TB drugs have been over $80 \%[5,6]$, but few studies evaluated the prediction of MDR and XDR based on WGS.

Therefore, in the current study, we performed WGS on drug-resistant M. tuberculosis isolates from China and Russia. One of our goals was to detect MDR and XDR based on limited numbers of mutation sites, and construct some combinations of drug-resistance-associated loci for predicting MDR/XDR with higher sensitivity and specificity at the same time. Another goal was to characterise the underlying drug-resistance-conferring mutations and higher-frequency novel mutations, promoting a comprehensive understanding of the mechanisms of drug-resistant $\mathrm{TB}$ and providing more information for clinical management.

\section{Materials and methods}

\section{Sample collection and processing}

A total of 105 M. tuberculosis clinical isolates were randomly sampled from among those collected from Shanghai Pulmonary Hospital, the biggest designated TB hospital in Shanghai 
City, between May 2017 and March 2018. These samples included 35 XDR cases, 35 MDR cases and 35 pan-susceptible cases.

According to the principle of equidistance, we selected the samples on the basis of the strains in the overall unit. MDR-TB indicates TB that is resistant to at least isoniazid (INH) and rifampicin (RIF), whereas XDR-TB indicates TB that is resistant not only to INH and RIF, but also to any fluoroquinolone and at least one of three injectable drugs, amikacin (AMI), kanamycin (KAN) and capreomycin (CAP). Drug susceptibility tests were performed by laboratory technologists from the TB laboratory using a fully automated Mycobacterial Growth Indicator Tube (MGIT) 960 liquid culture system (Becton Dickinson, USA). The critical concentrations of anti-TB drugs in these specific assays were as follows: rifampicin, $1.0 \mu \mathrm{g} / \mathrm{ml}$ (MGIT); isoniazid, $0.1 \mu \mathrm{g} / \mathrm{ml}$ (MGIT); ethambutol, 5.0 $\mu \mathrm{g} / \mathrm{ml}$ (MGIT); streptomycin, $1.0 \mu \mathrm{g} / \mathrm{ml}$ (MGIT); ofloxacin, $1.5 \mu \mathrm{g} /$ $\mathrm{ml}$ (MGIT); capreomycin, $2.5 \mu \mathrm{g} / \mathrm{ml}$ (MGIT); and amikacin, 1.0 $\mu \mathrm{g} / \mathrm{ml}$ (MGIT) [7]. All experimental methods conformed to standardised clinical laboratory procedures and operating regulations. DNA was extracted using a Mag-MK Bacterial Genomic DNA extraction kit according to the instructions provided. Quality control criteria for WGS included a minimum concentration $>50 \mathrm{ng} / \mu \mathrm{l}$ and a minimum $\mathrm{OD}_{260} / 280$ ratio (DNA purity) $>1.8$.

Because some samples failed to meet these criteria owing to failure of seeding, contamination of culture medium or extraction of DNA, only 97 isolates (30 XDR, $32 \mathrm{MDR}$ and 35 pansusceptible) were included in the study. Other isolates, including three MDR, 16 DR (resistant to any anti-TB drug other than XDR or MDR) and 21 pan-susceptible strains, were from Shanghai Minhang District Central for Disease Control and Prevention and Shanghai Putuo District Center for Disease Control and Prevention. The collection of these latter isolates was communitycentred, whereas the original 105 strains were obtained from the designated TB hospital. We also downloaded some genome sequences from the National Center for Biotechnology Information for some foreign strains in fastq file format, including 12 XDR cases, 39 MDR cases and 27 pan-susceptible cases. These strains were mainly derived from Francis Coll's [8] study of TB patients in Russia between 2008 and 2010. By inquiring the phenotypic drug sensitivity results of these strains and matching the corresponding original sequencing sequences, we finally included the sequences of 78 foreign strains in total.

\section{Sequencing}

WGS was performed on an Illumina HiSeq 2000 platform. Sickle (http://github.com/ucdavis-bioinformatics/sickle) was used for trimming reads, with a minimum base quality of Q20. Bowtie 2 [9], version 2.3.3, was used for mapping to the reference genome, M. tuberculosis H37RV, (NC_000962.3), with a minimum mapping quality of Q30. SAMtools [10], version 1.6, was used for calling single-nucleotide polymorphisms (SNPs), and VarScan (version 2.3.9) [11] was used for calling mutation variants. Sequencing errors and false positives were excluded based on a series of thresholds, including reference coverage $>95 \%$ and an average read depth $>20$. Mutation frequencies in cases where alternative alleles were based on fewer than $25 \%$ of reads were filtered using VarScan. Finally, SNPs located in Pro-Glu (PE) and Pro-Pro-Glu (PPE) regions, insertion elements, or repetitive regions were excluded.

\section{Identification of drug-resistance-conferring mutations}

A total of $215 \mathrm{M}$. tuberculosis isolates were obtained for genome sequencing, of which 97 were from Shanghai pulmonary hospital,
40 were from Shanghai Minhang District Central Hospital and Shanghai Putuo District Center for Disease Control and Prevention, and 78 were from Russia. Common drug-resistanceconferring mutant genes and their mutation frequencies may differ between domestic isolates and foreign isolates. We first selected 18 candidate genes for our drug-resistance mutation analysis process, taking into account those that were reported more than once in the scientific literature [12]. We found resistance mutations in only eight of these genes, possibly owing to the limited numbers of samples and/or lower mutation frequency. We presumed that synonymous and lineage-defining mutations were attributable to benign mutations that do not lead to a resistant phenotype. Since some mutations are only present in phenotypically susceptible strains or susceptibility is retained despite the presence of a mutation, mutations in all strains that were phenotypically susceptible were considered benign mutations. For all candidate genes, a mutation that occurred in at least one phenotypically resistant isolate was defined as resistance determining. Sometimes a resistant phenotype was not accounted for by a single resistance-conferring mutation in a candidate gene for a given drug as a result of synergy or co-occurring mechanisms among mutations [12]. In such cases, genome sequences were manually examined. An uncharacterised mutation corresponds to a mutation that occurred in a single phenotypically resistant isolate, and thus may not be a resistance-conferring mutation. The sensitivity of prediction was calculated by dividing the number of strains with drug phenotypic resistance mutations found by WGS in phenotypic resistance strains by the total number of phenotypic resistance strains. Fisher's exact test used to determine statistical difference of resistance mutations for each drug between different groups was performed in $\mathrm{R}$ (v4.0.2).

\section{Bootstrap analysis}

First, Perl scripts were used to combine SNP sites of all strains. Second, high-frequency resistance sites for each drug were filtered and combined in R (v4.0.2). Finally, a Bootstrap analysis (1000 times) was performed in $\mathrm{R}$ (v4.0.2) on select combinations to predict MDR and XDR isolates, calculating sensitivity and specificity and $95 \%$ confidence intervals (CI).

\section{Results}

\section{Characteristics of the samples}

We selected a total of 215 isolates for our genetic mutation analysis; 137 strains were collected from Shanghai, including 35 MDR, $30 \mathrm{XDR}, 16 \mathrm{DR}$ and 56 pan-susceptible strains. An additional 78 strains were from Russia isolates, including 39 MDR, $12 \mathrm{XDR}$ and 27 pan-susceptible strains. Online Supplementary Table S1 details drug-resistance information for patients. Four first-line drugs, rifampicin (RIF), isoniazid (INH), streptomycin (STR) and ethambutol (EMB), and three second-line drugs, amikacin (AMK), capreomycin (CAP) and ofloxacin (OFL), were considered in our analysis. Of these 215 isolates, 120 (55.8\%), 124 (57.7\%), 67 (31.2\%), 90 (41.9\%), 42 (19.5\%), 42 (19.5\%) and $42(19.5 \%)$ were resistant to RIF, INH, EMB, STR, OFX, AMK and CAP, respectively. A phylogenetic tree was constructed from a total of $215 \mathrm{M}$. tuberculosis strains using a maximum likelihood phylogenetic method in RAxML software (1000 bootstrap samples), yielding 162935 high-quality SNPs (Fig. 1). Alternative alleles identified in fewer than $25 \%$ of reads were filtered out, and 


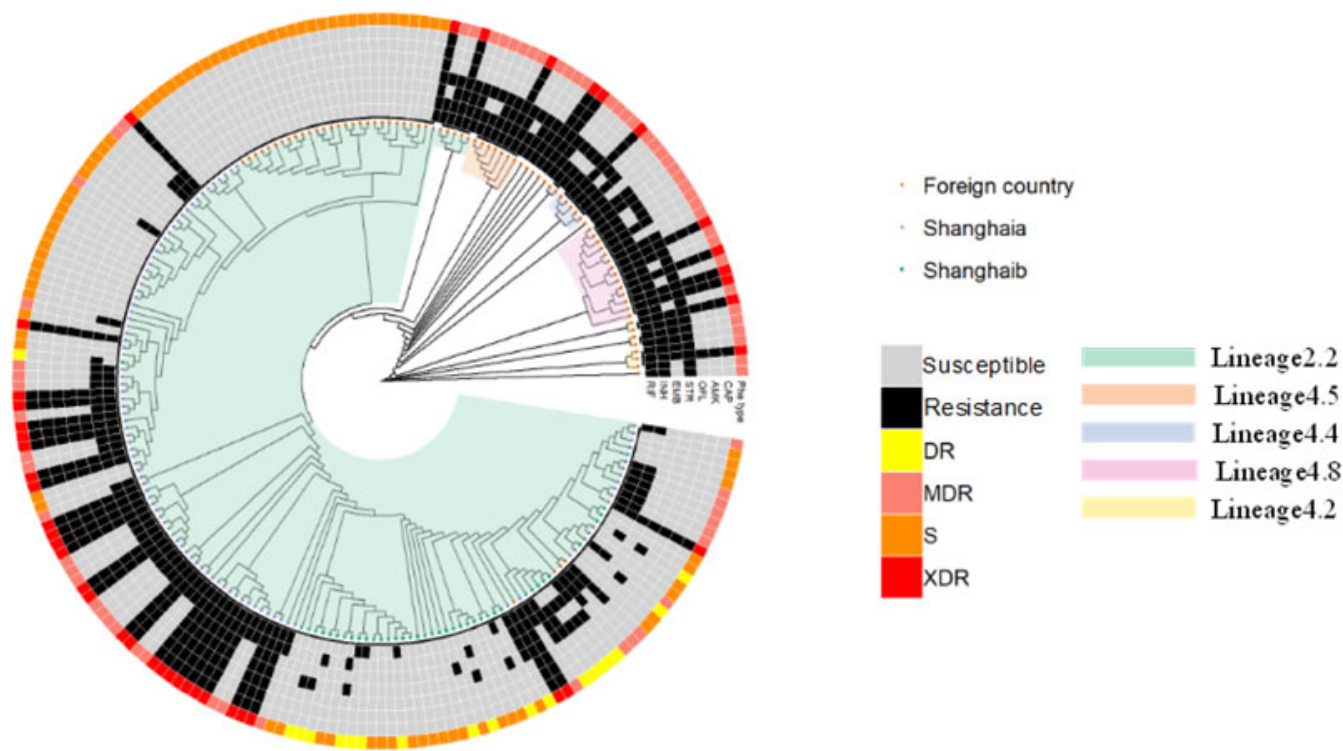

Fig. 1. Whole-genome phylogeny of the 215 M. tuberculosis isolates. Maximum likelihood phylogenetic tree (bootstrap $=1000$ times) constructed using 162936 SNPs spanning the whole genome and rooted on Mycobacterium canetti (not shown). Drug-resistance type is indicated by different colours in the outer ring of the circle; lineage definitions are indicated in the inner part of the circle; and drug-resistance profiles are shown in the middle portion of the circle. Russia isolates were randomly selected from among strains downloaded from GenBank. ' $S$ ' denotes isolates that were susceptible to all anti-TB drugs tested. 'DR' refers to isolates other than MDR or XDR strains that were resistant to any TB drugs. Shanghaia indicates that isolates are from Shanghai Pulmonary Hospital, Shanghaib indicates that isolates are from Shanghai Putuo and Minghang District Center for Disease Control and Prevention.

SNPs in PE/PPE regions, insertion elements and repeat regions were excluded from the analysis [13]. SNPs with a pairwise genetic distance of 12 or fewer SNPs were defined as recent transmissions [14]. No recent transmission events were present in the tree as finally constructed. We defined the lineage of strains using regions of difference [15]. A total of 191 strains (88.8\%), including $41 \mathrm{XDR}, 62 \mathrm{MDR}, 13 \mathrm{DR}$ and 75 pan-susceptible isolates, belonged to lineage 2.2 ('ancient' Beijing); 24 strains (11.2\%), containing one XDR, $12 \mathrm{MDR}$, three DR and eight pan-susceptible isolates, belonged to four sublineages, L4.2, L4.4, L4.5 and L4.8, designated 'Euro-American' lineages [16]. We found 30 drug-resistance markers in eight candidate genes among our 215 M. tuberculosis strains, as summarised in Table 1.

\section{Resistance to the first-line anti-TB medicines}

Mycobacterium tuberculosis strains that showed resistance to RIF generally exhibited mutations in the 81-bp $r p o B$ RIF-resistancedetermining region [17]. The most prevalent drug-resistanceconferring mutation was $r p o B$ Ser450Leu. Among domestic isolates, 69 strains were resistant to RIF, of which 42 strains, including $22 \mathrm{XDR}, 17 \mathrm{MDR}$ and three DR strains, possessed $r p o B$ Ser450Leu missense mutation. Among 56 pan-susceptible isolates, none resistance mutations were characterised. The sensitivity and specificity of $r p o B$ Ser450Leu in predicting phenotypic resistance to RIF were $60.87 \%$ and $100 \%$, respectively. Other strains lacking this mutation exhibited a lower frequency of other missense mutations. Among domestic 30 XDR strains, there were two isolates with an $r p o B$ Asp435Val mutation, one isolate with an $r p o B$ Gln172Arg mutation, one isolate with an $r p o B$ Gln432Lys mutation, one isolate with an $r p o B$ Gln432Pro mutation, and three phenotypically resistant strains without any mutation in the $r p o B$ gene. Among 35 MDR strains, there were three isolates with an $r p o B$ His445Pro mutation, two isolates with an $r p o B$ Asp435Val mutation, one isolate with an $r p o B$ Gln432Pro mutation, one isolate with an $r p o B$ His445Asp mutation, one isolate with co-occurrence of $r p o B$ His445Asp and His445Pro mutations, as well as phenotypically resistant strains without any mutations in the $r p o B$ gene. Among 16 DR strains, there were four phenotypically resistant strains without any mutations. Among Russia isolates, 47 drug-resistant strains carried an $r p o B$ Ser450Leu mutation. The sensitivity and specificity of rpoBSer450Leu in predicting phenotypic resistance to RIF were $92.16 \%$ and $100 \%$, respectively. There was also one strain with an $r p o B$ His445Asp mutant, and three phenotypically resistant strains without any mutations. Totally, WGS was capable of accounting for $79.71 \%(55 / 69)$ of phenotypically RIF-resistant domestic strains and $94.12 \% \quad(48 / 51)$ of phenotypically RIF-resistant Russia strains.

INH is a prodrug that is activated by the catalase-peroxidase enzyme $k a t G$, encoded by the kat $G$ gene [18]. Among our 215 M. tuberculosis strains, 124 exhibited resistance to INH. The most common drug-resistance-conferring mutation was kat $G$ Ser315Thr, in which the codon change may be G-C. Among domestic isolates, 52 strains carried a kat $G$ Ser315Thr mutation, including $26 \mathrm{MDR}, 21 \mathrm{XDR}$ and five DR strains. The sensitivity and specificity of kat $\mathrm{Ser} 315 \mathrm{Thr}$ in predicting phenotypic resistance to INH were $71.23 \%$ and $100 \%$, respectively. Among 30 XDR strains, there were 21 resistant strains with a katG Ser315Thr mutation, two strains with an inhA Ile21Thr mutation, one strain with an inhA Ser94Ala mutation, one strain with an $n d h$ Ala352Ser mutation, one strain with a katG Ala144Val mutation, one strain with a katG His97Arg mutation, and three strains that were phenotypically resistant to INH that had no mutations in our candidate genes. Among $35 \mathrm{MDR}$ strains, there were 26 strains with a katG Ser315Thr mutation, two strains with a katG Glu607Lys mutation, one strain with an inhA Ile21Thr mutation, one strain with a $k a t G$ Ala144Valmutation, one strain 
Table 1. Mutations identified within loci associated with resistance to anti-TB drugs in 215 clinical isolates

\begin{tabular}{|c|c|c|c|c|c|c|c|c|c|c|}
\hline \multirow[b]{2}{*}{ Drug } & \multirow[b]{2}{*}{$\begin{array}{l}\text { Gene } \\
\text { name }\end{array}$} & \multirow[b]{2}{*}{ Mutation } & \multicolumn{2}{|c|}{ Domestic isolates (137) } & \multirow[b]{2}{*}{$\begin{array}{l}\text { No.(\%) of } \\
\text { isolates } \\
\text { identified in } 16 \\
\text { DR strains }\end{array}$} & \multirow[b]{2}{*}{$\begin{array}{l}\text { No.(\%) of } \\
\text { isolates } \\
\text { identified in } 56 \\
\mathrm{~S}^{\mathrm{a}} \text { strains }\end{array}$} & \multirow{2}{*}{$\begin{array}{l}\text { Russia isolates } \\
\text { (78) } \\
\text { No.(\%) of } \\
\text { isolates } \\
\text { identified in } 39 \\
\text { MDR strains }\end{array}$} & \multirow[b]{2}{*}{$\begin{array}{l}\text { No.(\%) of } \\
\text { isolates } \\
\text { identified in } 12 \\
\text { XDR strains }\end{array}$} & \multirow[b]{2}{*}{$\begin{array}{c}\text { No.(\%)of } \\
\text { isolates } \\
\text { identified in } 27 \\
\text { S strains }\end{array}$} & \multirow[b]{2}{*}{$P$ value } \\
\hline & & & $\begin{array}{l}\text { No. (\%) of } \\
\text { isolates } \\
\text { identified in } 35 \\
\text { MDR strains }\end{array}$ & $\begin{array}{l}\text { No.(\%) of } \\
\text { isolates } \\
\text { identified in } 30 \\
\text { XDR strains }\end{array}$ & & & & & & \\
\hline RIF & rpoB & S450L & $17(48.57)$ & 22 (73.33) & $3(75.00)$ & - & $37(94.87)$ & $10(83.33)$ & - & $>0.05$ \\
\hline RIF & rрoв & D435V & $2(5.71)$ & $2(6.67)$ & - & - & - & - & - & \\
\hline RIF & rpoB & $\mathrm{H} 445 \mathrm{D} / \mathrm{P}$ & $5(14.29)$ & - & - & - & $1(2.56)$ & - & - & \\
\hline RIF & rpoB & Q172R & - & $1(3.33)$ & - & - & - & - & - & \\
\hline RIF & rрoв & $\mathrm{Q} 432 \mathrm{P} / \mathrm{K}$ & $1(2.86)$ & $2(6.67)$ & - & - & - & - & - & \\
\hline INH & katG & S315T & $26(74.29)$ & $21(70.00)$ & $5(62.50)$ & - & $37(94.87)$ & $10(83.33)$ & - & $>0.05$ \\
\hline INH & katG & A144V & $1(2.86)$ & $1(3.33)$ & - & - & - & - & - & \\
\hline INH & katG & $\mathrm{H} 97 \mathrm{R}$ & - & $1(3.33)$ & - & - & - & - & - & \\
\hline $\mathrm{INH}$ & katG & E607K & $2(5.71)$ & - & - & - & - & - & - & \\
\hline INH & katG & S17G & $1(2.86)$ & - & - & - & - & - & - & \\
\hline INH & inhA & $121 \mathrm{~T}$ & $1(2.86)$ & $2(6.67)$ & - & - & - & - & - & \\
\hline $\mathrm{INH}$ & inhA & S94A & - & $1(3.33)$ & - & - & - & - & - & \\
\hline INH & ndh & A352S & - & $1(3.33)$ & - & - & - & - & - & \\
\hline STR & rpsL & $\mathrm{K} 43 \mathrm{R}$ & $2(5.88)$ & $17(56.7)$ & $8(47.06)$ & - & $8(20.51)$ & $6(50.00)$ & - & $>0.05$ \\
\hline STR & rpsL & $\mathrm{K} 88 \mathrm{R}$ & - & $5(16.67)$ & $2(11.76)$ & - & $1(2.56)$ & - & - & \\
\hline STR & rrs & $\mathrm{C} 517 \mathrm{~T}$ & - & 4 (13.33) & - & - & - & $3(25.00)$ & - & \\
\hline EMB & embB & M306V & - & $12(40.00)$ & $1(5.88)$ & & $6(28.57)$ & $3(25.00)$ & - & $<0.05$ \\
\hline EMB & embB & M306L & - & - & $1(5.88)$ & - & - & - & - & \\
\hline EMB & embB & D354A & - & - & - & - & $16(76.19)$ & $3(25.00)$ & - & \\
\hline EMB & embB & M306I & - & $5(16.67)$ & - & - & - & - & - & \\
\hline EMB & embB & Q497R & - & $2(6.67)$ & - & - & - & $1(8.33)$ & - & \\
\hline EMB & embB & G406A & - & $3(10.00)$ & - & - & - & - & - & \\
\hline EMB & embB & G406S & - & $2(6.67)$ & - & - & - & - & - & \\
\hline OFL & gyrA & D94G & - & $10(33.33)$ & - & - & - & $2(16.67)$ & - & $>0.05$ \\
\hline OFL & gyrA & A90V & - & $9(30.00)$ & - & - & - & - & - & \\
\hline OFL & gyrA & D94A & - & $3(10.00)$ & - & - & - & - & - & \\
\hline OFL & gyrA & D94N & - & $3(10.00)$ & - & - & - & $1(8.33)$ & - & \\
\hline AMK/CAP & rrs & A1401G & - & 19 (63.33) & - & - & - & $3(25.00)$ & - & $>0.05$ \\
\hline AMK/CAP & rrs & C1402T & - & $1(3.33)$ & - & - & - & $1(8.33)$ & - & \\
\hline AMK/CAP & rrs & G1484T & - & $1(3.33)$ & - & - & - & - & - & \\
\hline
\end{tabular}

RIF, rifampicin; INH, isoniazid; EMB, ethambutol; STR, streptomycin; AMK, amikacin; CAP, capreomycin; OFL, ofloxacin.

${ }^{a} S$ isolate indicates pan-susceptible isolate (i.e. sensitive to four first-line anti-TB drugs). 
with a katG Ser17Gly mutation, and one strain that carried no mutations. Among eight DR strains, there were five strains that carried a kat G Ser315Thr mutation. Among Russia isolates, 10 XDR and 37 MDR strains carried a katG Ser315Thr mutation. The frequency of the katG Arg463Leu mutation, used as a phylogenetic marker, was higher than that of any other mutation among our selected genes, but because it does not confer INH resistance, it was excluded. WGS was capable of predicting $86.30 \%(63 / 73)$ of phenotypically INH-resistant domestic strains and $92.16 \%(47 / 51)$ of phenotypically INH-resistant Russia strains.

Mutations in the rpsL gene, encoding a $30 \mathrm{~S}$ ribosomal protein associated with the first step of RNA translation, have been reported to account for approximately $80 \%$ of STR resistance [19]. Thus, the rpsL gene was selected as a candidate gene for assessing STR resistance in different strains. The most frequently detected mutation was rpsL Lys43Arg, which was present in 41 phenotypically STR-resistant strains. The sensitivity and specificity of the rpsL Lys43Arg mutation in predicting phenotypic STR resistance were $45.56 \%$ and $100 \%$, respectively. Among domestic isolates, there were $17 \mathrm{XDR}$ strains with the $r p s L$ Lys43Arg mutation, five XDR strains with the rpsL Lys88Arg mutation, eight DR strains with the rpsL Lys43Arg mutation, and two DR strains with the rpsL Lys88Arg mutation. Among Russia isolates, there were eight MDR and six XDR strains with the rpsL Lys43Arg mutation and one MDR strain with the rpsL Lys88Arg mutation. There were also detected rrs C517T mutation in four XDR domestic isolates and three XDR Russia isolates. WGS was capable of predicting $88.37 \%$ (38/43) of phenotypically STR-resistant domestic strains and $38.30 \%$ (18/47) of phenotypically STR-resistant Russia strains.

Drug-resistance-conferring mutations associated with EMB primarily occur in the $e m b B$ gene [20]. The most common mutation in our phenotypically EMB-resistant strains was embB Met306Val; among 67 phenotypically resistant isolates, 22 strains carried this mutation. Among domestic isolates, there were 12 XDR strains with an $e m b B$ Met306Val mutation, five XDR strains with an $e m b B$ Met306Ile mutation, two XDR strains with an $e m b B$ Gln497Arg mutation, three XDR strains with an $e m b B$ Gly406Ala mutation, and two XDR strains with an embB Gly406Ser mutation. Among two DR strains that were phenotypically resistant to EMB, one strain carried an embB Met306Val mutation and one strain carried an $e m b B$ Met306Leu mutation. Among Russia isolates, six MDR and three XDR strains carried an $e m b B$ Met306Val mutation, $16 \mathrm{MDR}$ and three XDR strains carried an $e m b B$ Asp354Ala mutation, and one XDR strain carried an $e m b B$ Gln497Arg mutation. WGS was capable of predicting $76.47 \%(26 / 34)$ of phenotypically EMB-resistant domestic strains and $87.88 \%(29 / 33)$ of phenotypically EMB-resistant Russia strains.

\section{Mutations related to the second-line drugs}

Mutations in the rrs gene, encoding 16S rRNA, are markers associated with AMK and CAP resistance, especially mutations occurring at nucleotide positions 1401, 1402 and 1484 [21]. Among 30 XDR domestic strains, the most prevalent AMK/ CAP-resistance-conferring mutation was $\operatorname{rrs}$ A1401G, which was found in 19 phenotypically resistant strains. The sensitivity and specificity of the rrs A1401G mutation in predicting phenotypic resistance to AMK/CAP were $63.33 \%$ and $100 \%$, respectively. Other mutations detected among XDR domestic strains were rrs
C1402T and rrs G1484T, found in one strain each. Among 12 Russia XDR isolates, there were three with an rrs A1401G mutation, one with an $r$ rs C1402T mutation. WGS was capable of predicting $70.00 \%(21 / 30)$ of phenotypically AMK/CAP-resistant domestic strains and $33.33 \%(4 / 12)$ of phenotypically AMK/ CAP-resistant Russia strains.

Resistance to OFL is associated with mutations in the genes, gyr $A$ and $g y r B$, encoding subunits that constitute the heterotetrameric protein, DNA gyrase; these mutations occur predominantly at codons 88-94 [22]. Among 30 domestic XDR strains, the most common drug-resistance-conferring mutation was gyrA Asp94Gly, which was present in 10 phenotypically OFL-resistant strains. The sensitivity and specificity of the gyrA Asp94Gly mutation in predicting phenotypic resistance to OFL were $33.33 \%$ and $100 \%$, respectively. Other mutations included gyrA Ala90Val, found in nine phenotypically resistant strains; gyrA Asp94Ala, found in three strains; and gyrA Asp94Asn, found in three strains. Among 12 Russia XDR strains, two strains carried a gyrA Asp94Gly mutation and one strain carried a gyrA Asp94Asn mutation. WGS was capable of predicting $83.33 \%(25 / 30)$ of phenotypically OFL-resistant domestic strains and $25.00 \%(3 / 12)$ of phenotypically OFL-resistant Russia strains.

\section{Prediction value of loci combination by bootstrap}

Finally, we used a bootstrap approach (1000 times) to create combinations of highly prevalent drug-resistance locations to predict the XDR/MDR-resistant phenotype. The sensitivity and specificity of our combinations are summarised in Table 2 . The highest sensitivity with respect to the prediction of MDR against RIF and INH was $92.17 \%(0.8615,0.9646)$, which was provided by the combination of $r p o B \mathrm{~S} 450 \mathrm{~L}+r p o B \mathrm{H} 445 \mathrm{~A}+r p o B \mathrm{H} 445 \mathrm{P}+k a t G$ $\mathrm{S} 315 \mathrm{~T}+i n h A \mathrm{I} 21 \mathrm{~T}+i n h A \mathrm{~S} 94 \mathrm{~A}$ mutations. The highest sensitivity with respect to the prediction of XDR against RIF, INH, AMK/ CAP and OFL was $92.86 \%(0.8158,0.9796)$, which was provided by the combination of $r p o B$ S450L + katG S315T + gyrA D94G + rrs A1401G mutations. We also tested the inclusion of additional mutations in combinations, but none of these additions improved the predictive effect.

\section{Discussion}

We found that WGS was capable of predicting about $82.07 \%$ and $72.33 \%$ of phenotypic drug resistance for China and Russia strains, respectively. For domestic strains, the predictive sensitivity from the highest to the lowest was STR $(88.37 \%)$, INH $(86.30 \%)$, OFL (83.33\%), RIF (79.71\%), EMB (76.47\%) and AMK/CAP (70\%). For Russia strains, the sequence was RIF (94.12\%), INH (92.16\%), EMB (87.88\%), STR (38.30\%), AMK/CAP (33.33\%) and OFL (25.00\%). First, geographic variation may explain the differences of mutant frequency in predictive values. Second, the predictive sensitivity of STR resistance was the best in China using the WGS method, which could be due to the fact that STR resistance-conferring mutations were largely found in China. Finally, the sensitivity of WGS in detecting resistance to second-line drugs were unsatisfactory, possibly because of the limited numbers of isolates, preventing us from discovering the full variety of mutations.

The phylogenetic tree showed that lineage 2 and lineage 4 spread in both Russia and China, and L2.2 constituted the predominant strain, accounting for $97.4 \%(76 / 78)$ in Russia and $83.9 \%(115 / 137)$ in China. The fact that $M$. tuberculosis lineage 


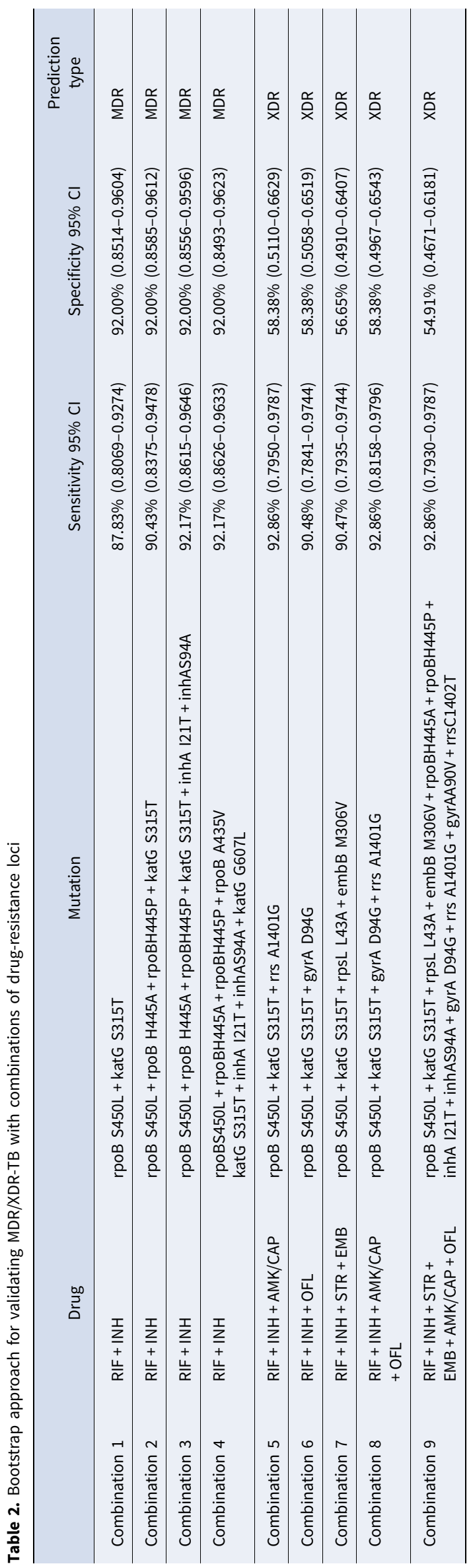

2.2, also called Beijing family, is equipped with higher adaptability, resistance and virulence may be responsible for its widely prevalence. Actually, human-adapted M. tuberculosis complex comprises seven lineages of various geography distributions. Lineage 2 and lineage 4 distributed in worldwide, while other lineages exhibit a geographical restriction. Moreover, susceptible, drug-resistant and MDR strains existed in lineage 2 and lineage 4, but XDR strains almost exclusively existed in lineage 2.2 , which demonstrates that different lineages may play a different role in the outcome of drug resistance.

Only eight resistance genes among 18 candidate genes, and a total of 30 mutations of these eight resistance genes were identified, possibly attributing to a lack of sufficient samples and low rate of mutation. Limited numbers of samples lack the ability to identify comprehensive mutations. Among 30 detected mutations, 26 mutations have been reported in previous studies and four mutations were novel mutations ( $r p o B$ Q172R, katG A144V, kat $\mathrm{G} \mathrm{H97R,} \mathrm{katG} \mathrm{S17G),} \mathrm{but} \mathrm{whether} \mathrm{it} \mathrm{could} \mathrm{be} \mathrm{regarded} \mathrm{as} \mathrm{mar-}$ kers of drug resistance remains to be further studied. In addition, we identified $k a t G$ Arg463Leu, gyrA Glu21Gln, gyrA Ser95Thr and gyrA Gly668Asp as lineage-defining mutations. These mutations, which have been reported to be natural polymorphisms that are useful for evolutionary characterisation of the genome [23], were present at elevated frequencies in both resistant and sensitive strains, and thus did not represent drug-resistance-conferring mutations.

Although the specificity of representative genes in predicting phenotype was $100 \%$, it could be overestimated because we assumed that strains without detecting corresponding mutations were susceptible. The rates of dominant mutations of seven drugs in this study were INH (71.23\%), RIF (60.87\%), STR (62.79\%), EMB (38.24\%), AMK/CAP (63.33\%) and OFL (33.33\%). About other mutations of each drug are discussed in the following. For rifampicin resistance, in addition to those phenotypic resistance without any mutation, the rest RIF-resistance-conferring mutations occurred exclusively in the $r p o B$ gene region in this study, including $r p o B$ Ser450Leu, $r p o B$ Asp435Val, rpoB His445Asp/Pro and $r p o B$ Gln432Pro/Lys which have been reported in previous studies [24-26]. We also found a novel mutation, $r$ poB Gln $172 \mathrm{Arg}$, but it was only detected in a single resistant isolate. The kat $G$ gene is thought to have a high association with INH resistance and the most frequently reported katG mutation, Ser315Thr, was found in this study. Other previously known mutations, including inhA Ile21Thr and inhA Ser94Ala, were also identified $[27,28]$. We also identified three novel mutations, katG Ala144Val, katG His97Arg and $k a t G$ Ser17Gly, but further studies are needed to elucidate whether these mutations really confer resistance to INH or not. The most commonly reported mutation of EMB resistance, $e m b B$ Met306Val, was identified in the current study and other previously reported mutations [29, 30], embB Gln497Arg, embB Gly406Ala and $e m b B$ Asp354Ala, also were identified. Nevertheless, identifying embB Met306Val mutation to predict phenotype was not an effective option since the predictive sensitivity was quite lower than expected, which reminds us that $e m b B$ Met306Val mutation may be just one small part of all main EMB resistance-conferring mutations. Other candidate genes and mutation sites of EMB resistance need to be further explored. STR resistance-conferring mutations mainly happen in the $r p s L$ and $r r s$ genes [31]. However, some related studies have indicated that the association of mutations in the rrs gene with resistance is not obvious [32], instead reporting that mutations of lysine to 
arginine in the rpsL gene are highly correlated with the development of STR resistance. In our results, the most frequent mutations in $r p s L$ gene were $r p s L$ Lys43Arg and $r p s L$ Lys88Arg, similar to the results of Spies et al. [33]. For OFL-resistant strains, two mutations with high frequency, gyrA Ala90Val and gyrA Asp94Gly, that were reported previously [34] were also found in this study, additional mutations including gyrA Asp94Ala and gyrA Asp94Asn were also found in this study, and both of them have been reported [35]. Cross-resistance to AMK and CAP attributes to mutations in the rss gene, with the mutations rrs A1401G, rrs C1402T and rrs G1484T being the most common [36]. In our study, we found that rrs A1401G, detected in $63.3 \%$ of AMK/CAP-resistant isolates, was the most prevalent. The frequency of other mutations was lower than expected.

In the attempt to improve the predictive power of WGS, we applied a bootstrap approach to identify combinations of highfrequency mutations that might prove more sensitive. For predicting the MDR phenotype, we found that the lowest predictive sensitivity combination was $r p o B$ S450L $+k a t G$ S315T. And the predictive sensitivity increased with including more mutation sites, with the highest predictive sensitivity combination of $r p o B$ $\mathrm{S} 450 \mathrm{~L}+r p o B \mathrm{H} 445 \mathrm{~A} / \mathrm{P}+$ katG S315T + inhA I21T + inhA S94A. No obvious improvement on MDR predictive sensitivity was observed when $r p o B$ A435V and KatG G607L mutations were added. For predicting XDR phenotype, the mutation combination with highest sensitivity (92.86\%) was rpoB S450L + katG S315T + gyrA D94G + rrs A1401G. Similarly, no significant improvement was observed when adding more mutations. WGS, in recent years, has been increasingly used to predict drug resistance and guide drug-susceptibility testing patterns. Several automated software tools have been developed for this, such as CASTB, KvarQ, Mykrobe Predictor TB, PhyResSE and TB Profiler [37]. Most studies indicated that WGS has a high sensitivity to predict isoniazid and rifampicin resistance prediction, but a variable performance for resistance prediction of other drugs. This might be due to the incompleteness of drug-resistance profiles in local. While most resistance prediction focused on first-line drugs or single drug, hence, the combinations of resistance prediction in present study might provide useful references for MDR and XDR prediction [38].

For resistance rates of seven drugs in Russia and China, the proportion of resistance to RIF and INH accounted for the vast majority while the percentage of resistance to second-line drug (OFL) and injectable drugs (AMK/CAP) were quite low, which are consistent with most previous studies. In fact, resistance rates reflect the selective pressure of anti-TB drugs on the evolution of M. tuberculosis and the long-term anti-TB treatment leads to the continuous emergence and expansion of resistant strains. For resistance phenotype, the proportion of MDR-TB in Russia $(50.0 \%)$ was much higher than in domestic (23.4\%), probably because of the history of high level of MDR-TB in Russian federation. The difference in resistance rates and phenotype implied that resistance-conferring mutations are highly associated with geographic location.

WGS, on the one hand, offers a number of advantages compared with traditional DST [39, 40], which reduce diagnostic time to 4-8 days compared with traditional laboratory culture methods. The current cost of diagnosing TB with WGS is about $\$ 80$ per case, which is actually slightly less than the cost of DST. On the other hand, WGS certainly has some limitations. For example, it could not show the minimum inhibitory concentration of drug resistance compared with DST and there are some phenotypically drug-resistant isolates without any gene mutation. One of the limitations in this study is the selection bias of strains and insufficient resistance candidate genes, which reflects the relatively low predictive sensitivity of WGS for some drug. In addition, the definition of XDR-TB has changed in January 2021 [41], which deserves further observation and adjustment in DST prediction.

\section{Conclusions}

We found that WGS has a higher sensitivity and specificity in predicting resistance to first-line anti-TB drugs, compared with second-line drugs. We also identified some novel mutations in $k a t G$ and $r p o B$ genes, but additional insight into drug-resistance mechanisms is needed. These novel mutations, together with frequent mutations, can provide a reference for clinical microbiology laboratory diagnostic methods for identifying drug-resistant TB. The valuable mutation combination, identified using the bootstrap method, for predicting MDR-TB phenotype was $r p o B$ $\mathrm{S} 450 \mathrm{~L}+r p o B \mathrm{H} 445 \mathrm{~A} / \mathrm{P}+k a t G \mathrm{~S} 315 \mathrm{~T}+i n h A \mathrm{I} 21 \mathrm{~T}+i n h A \mathrm{~S} 94 \mathrm{~A}$. For second-line drugs, and for predicting XDR-TB phenotype, it was $r p o B$ S450L + katG S315T + gyrA D94G + rrs A1401G.

Supplementary material. The supplementary material for this article can be found at https://doi.org/10.1017/S095026882100279X

Author contributions. H. X., W. W. and F. Y. designed this study. L. W. and J. Y. collected the M. tuberculosis isolates applied in this study. L. W., J. Y., L. C., W. W. and H. X. discussed the outcome of genome sequencing. L. W. and J. Y. performed the statistical analysis and wrote this manuscript. W. W. and H. X. revised the structure and language of this paper. All authors reviewed the manuscript and agreed to the published version of this manuscript.

Financial support. This study was granted by the National Key Scientific and Technological Project against Major Infectious Diseases (Grant nos. 2017ZX10201302-007-003 and 2018ZX10722302-005), and the Shanghai New Three-year Action Plan for Public Health (Grant no. GWV-10.1-XK16).

\section{Conflict of interest. None.}

Author statement. We declare that the work described was original research that has not been published previously, and not under consideration for publication elsewhere, in whole or in part. All the authors listed have approved the manuscript that is enclosed.

Data availability statement. Available on reasonable request.

\section{References}

1. Kerubo G et al. (2016) Drug susceptibility profiles of pulmonary Mycobacterium tuberculosis isolates from patients in informal urban settlements in Nairobi, Kenya. BMC Infectious Diseases 16, 583.

2. Helb D et al. (2010) Rapid detection of Mycobacterium tuberculosis and rifampin resistance by use of on-demand, near-patient technology. Journal of Clinical Microbiology 48, 229-237.

3. Hillemann D, Rüsch-Gerdes S and Richter E (2007) Evaluation of the GenoType MTBDRplus assay for rifampin and isoniazid susceptibility testing of Mycobacterium tuberculosis strains and clinical specimens. Journal of Clinical Microbiology 45, 2635-2640.

4. Hillemann D, Rüsch-Gerdes S and Richter E (2009) Feasibility of the GenoType MTBDRsl assay for fluoroquinolone, amikacin-capreomycin, and ethambutol resistance testing of Mycobacterium tuberculosis strains and clinical specimens. Journal of Clinical Microbiology 47, 1767-1772.

5. Faksri K et al. (2019) Comparisons of whole-genome sequencing and phenotypic drug susceptibility testing for Mycobacterium tuberculosis 
causing MDR-TB and XDR-TB in Thailand. International Journal of Antimicrobial Agents 54, 109-116.

6. Papaventsis D et al. (2017) Whole genome sequencing of Mycobacterium tuberculosis for detection of drug resistance: a systematic review. Clinical Microbiology and Infection 23, 61-68.

7. Katende B et al. (2020) Rifampicin resistant tuberculosis in Lesotho: diagnosis, treatment initiation and outcomes. Scientific Reports 10, 1917.

8. Coll F et al. (2018) Genome-wide analysis of multi- and extensively drug-resistant Mycobacterium tuberculosis. Nature Genetics 50, 307-316.

9. Langmead B and Salzberg SL (2012) Fast gapped-read alignment with Bowtie 2. Nature Methods 9, 357-359.

10. Li H et al. (2009) The sequence alignment/map format and SAMtools. Bioinformatics (Oxford, England) 25, 2078-2079.

11. Koboldt DC et al. (2012) VarScan 2: somatic mutation and copy number alteration discovery in cancer by exome sequencing. Genome Research 22, 568-576.

12. Walker TM et al. (2015) Whole-genome sequencing for prediction of Mycobacterium tuberculosis drug susceptibility and resistance: a retrospective cohort study. Lancet Infectious Diseases 15, 1193-1202.

13. Stamatakis A, Hoover $\mathbf{P}$ and Rougemont $\mathbf{J}$ (2008) A rapid bootstrap algorithm for the RAxML Web servers. Systematic Biology 57, 758-771.

14. Yang C et al. (2015) Transmission of Mycobacterium tuberculosis in China: a population-based molecular epidemiologic study. Clinical Infectious Diseases 61, 219-227.

15. Faksri K et al. (2016) In silico region of difference (RD) analysis of Mycobacterium tuberculosis complex from sequence reads using RD-Analyzer. BMC Genomics 17, 847.

16. Liu Q et al. (2018) China's tuberculosis epidemic stems from historical expansion of four strains of Mycobacterium tuberculosis. Nature Ecology \& Evolution 2, 1982-1992.

17. Heep M et al. (2001) Frequency of rpoB mutations inside and outside the cluster I region in rifampin-resistant clinical Mycobacterium tuberculosis isolates. Journal of Clinical Microbiology 39, 107-110.

18. Cade CE et al. (2010) Isoniazid-resistance conferring mutations in Mycobacterium tuberculosis KatG: catalase, peroxidase, and INH-NADH adduct formation activities. Protein Science 19, 458-474.

19. Chakraborty S et al. (2013) Para-aminosalicylic acid acts as an alternative substrate of folate metabolism in Mycobacterium tuberculosis. Science (New York, N.Y.) 339, 88-91.

20. Jagielski T et al. (2016) Methodological and clinical aspects of the molecular epidemiology of Mycobacterium tuberculosis and other mycobacteria. Clinical Microbiology Reviews 29, 239-290.

21. Bhembe NL et al. (2014) Molecular detection and characterization of resistant genes in Mycobacterium tuberculosis complex from DNA isolated from tuberculosis patients in the Eastern Cape province South Africa. BMC Infectious Diseases 14, 479.

22. Disratthakit A et al. (2016) Role of gyrB mutations in pre-extensively and extensively drug-resistant tuberculosis in Thai clinical isolates. Antimicrobial Agents and Chemotherapy 60, 5189-5197.

23. Lau RW et al. (2011) Molecular characterization of fluoroquinolone resistance in Mycobacterium tuberculosis: functional analysis of gyrA mutation at position 74. Antimicrobial Agents and Chemotherapy 55, 608-614.

24. Donnabella V et al. (1994) Isolation of the gene for the beta subunit of RNA polymerase from rifampicin-resistant Mycobacterium tuberculosis and identification of new mutations. American Journal of Respiratory Cell and Molecular Biology 11, 639-643.
25. Ramaswamy SV et al. (2004) Genotypic analysis of multidrug-resistant Mycobacterium tuberculosis isolates from Monterrey, Mexico. Journal of Medical Microbiology 53(Pt 2), 107-113.

26. Sajduda A et al. (2004) Molecular characterization of rifampin- and isoniazid-resistant Mycobacterium tuberculosis strains isolated in Poland. Journal of Clinical Microbiology 42, 2425-2431.

27. Leung ET et al. (2006) Molecular characterization of isoniazid resistance in Mycobacterium tuberculosis: identification of a novel mutation in inhA. Antimicrobial Agents and Chemotherapy 50, 1075-1078.

28. Morlock GP et al. (2003) ethA, inhA, and katG loci of ethionamide-resistant clinical Mycobacterium tuberculosis isolates. Antimicrobial Agents and Chemotherapy 47, 3799-3805.

29. Plinke C et al. (2010) embCAB sequence variation among ethambutolresistant Mycobacterium tuberculosis isolates without embB306 mutation. Journal of Antimicrobial Chemotherapy 65, 1359-1367.

30. Ramaswamy SV et al. (2000) Molecular genetic analysis of nucleotide polymorphisms associated with ethambutol resistance in human isolates of Mycobacterium tuberculosis. Antimicrobial Agents and Chemotherapy 44, 326-336.

31. Hlaing YM et al. (2017) Mutations in streptomycin resistance genes and their relationship to streptomycin resistance and lineage of Mycobacterium tuberculosis Thai isolates. Tuberculosis and Respiratory Diseases (Seoul) 80, 159-168.

32. Sun $\mathbf{H}$ et al. (2016) Characterization of mutations in streptomycinresistant Mycobacterium tuberculosis isolates in Sichuan, China and the association between Beijing-lineage and dual-mutation in gidB. Tuberculosis (Edinburgh) 96, 102-106.

33. Spies FS et al. (2011) Streptomycin resistance and lineage-specific polymorphisms in Mycobacterium tuberculosis gidB gene. Journal of Clinical Microbiology 49, 2625-2630.

34. Chen L, Zhang J and Zhang H (2016) Heteroresistance of Mycobacterium tuberculosis strains may be associated more strongly with poor treatment outcomes than within-host heterogeneity of $M$. tuberculosis infection. Journal of Infectious Diseases 214, 1286-1287.

35. Takiff HE et al. (1994) Cloning and nucleotide sequence of Mycobacterium tuberculosis gyrA and gyrB genes and detection of quinolone resistance mutations. Antimicrobial Agents and Chemotherapy 38, 773-780.

36. Maus CE, Plikaytis BB and Shinnick TM (2005) Molecular analysis of cross-resistance to capreomycin, kanamycin, amikacin, and viomycin in Mycobacterium tuberculosis. Antimicrobial Agents and Chemotherapy 49, 3192-3197.

37. Schleusener V et al. (2017) Mycobacterium tuberculosis resistance prediction and lineage classification from genome sequencing: comparison of automated analysis tools. Scientific Reports 7, 46327.

38. Allix-Béguec C et al. (2018) Prediction of susceptibility to first-line tuberculosis drugs by DNA sequencing. New England Journal of Medicine 379, 1403-1415.

39. Hang NTL et al. (2019) Whole genome sequencing, analyses of drug resistance-conferring mutations, and correlation with transmission of Mycobacterium tuberculosis carrying katG-S315T in Hanoi, Vietnam. Scientific Reports 9, 15354.

40. Takii T et al. (2019) Whole-genome sequencing-based epidemiological analysis of anti-tuberculosis drug resistance genes in Japan in 2007: application of the Genome Research for Asian Tuberculosis (GReAT) database. Scientific Reports 9, 12823.

41. World Health Organization (WHO). https://www.who.int/publications/i/ item/meeting-report-of-the-who-expert-consultation-on-the-definition-ofextensively-drug-resistant-tuberculosis (Accessed 23 October 2021). 